\title{
MONTE CARLO SIMULATIONS OF SEGREGATION AT [001] TWIST BOUNDARIES IN A Pt(Au) ALLOY-II. DISCUSSION
}

\author{
A. SEKI', D. N. SEIDMAN', Y. OH ${ }^{1}$ and S. M. FOILES ${ }^{2}$ \\ ${ }^{1}$ Northwestern University, Department of Materials Science and Engineering and the Materials Research \\ Center, Robert R. McCormick School of Engineering and Applied Science, Evanston, IL 60208-3108 and \\ ${ }^{2}$ Sandia National Laboratories, Theoretical Division, Livermore, CA 94551, U.S.A.
}

(Received 4 March 1991)

\begin{abstract}
Abetrect-A three dimensional plot of the density of Au atoms versus the position coordinates for the (002) planes, immediately adjacent to the interface, for a $\theta=5^{\circ}[001]$ twist boundary, suggests the presence of a hill-and-valley structure in the Au segregation behavior at $850 \mathrm{~K}$. The valleys correspond to the regions of good atomic fit between the regions of misfit; the hills correspond to the cores of the pairs of orthogonal primary grain boundary dislocations. A model for the Monte Carlo simulations is developed that leads to a linear expression for $S_{\text {aver }}$ containing a core segregation factor $\left(S_{\text {core }}\right)$. For this model $S_{\text {core }}$ has a single value for all twist boundaries at a given $T$. All the Monte Carlo results can be plotted on a single Arrhenius plot and the Gibbs free binding energy of a Au atom to the cores of the primary grain boundary dislocations extracted; the binding enthalpy and entropy of segregation are $0.095 \pm 0.01 \mathrm{eV}$ and $k_{\mathrm{B}}(0.49 \pm 0.10)$ respectively.
\end{abstract}

Reman-Un diagramme à trois dimensions de la densite d'atomes Au contre les positions coordonces pour les plans (002), immediatement adjacents à l'interface, pour un joint de grain vis [001] avec $\theta=5^{\circ}$, suggére la présence d'une structure de "monts et vaux" dans le comportement de ségrégation à $850 \mathrm{~K}$. Les "vaux" correspondent aux régions où les atomes s'harmonisent entre les régions de non-harmonie, les "monts" correspondent aux coeurs de paires perpendiculaires de dislocations primaire de joint de graines. Un modèle est développé pour les simulations Monte Carlo qui conduit à une expression linéaire pour $S_{\text {aver }}$ contenant un facteur de coeur de ségrégation $\left(S_{\text {core }}\right)$. Dans $\propto$ modèle, $S_{\text {core }}$ a une seule valeur pour tous les joints de grains torsion à un certain $T$. Ainsi, toutes les simulations Monte Carlo peuvent être tracés sur un seul diagramme Arrhenius de $S_{\text {core }}$ et l'énergie libre de liaison Gibbs d'un atome Au au coeur de dislocations de joints de grains primaires est determinée. L'enthalpie et entropie de liaison sont $0.095 \pm 0.01 \mathrm{eV}$ et $k_{\mathrm{B}}(0.49 \pm 0.10)$ respectivement.

\begin{abstract}
Zumammenfoum - Eine dreidimensionale Darstellung der Dichte von Au-Atomen über der Ortskoordinate in den die Grenzfläche einer [001]-Drillgrenze mit Drillwinkel $\theta=5^{\circ}$ beschreibenden beiden (002)-Ebenen legt nahe, daB im Au-Segregationsverhalten bei $850 \mathrm{~K}$ ein Berg- und Talverhalten vorliegt. Die Táler entsprechen den Bereichen guter Atompassung zwischen den fehlpassenden Bereichen. Die Berge entsprechen den Kernen von Paaren aus orthogonalen Korngrenzversetzungen. Für Monte-CarloSimulationen wird ein Modell entwickelt, welches zu einem linearen Ausdruck für $S_{\text {vurt }}$ führ den Kernegregationsfaktor $S_{\text {oore }}$ enthält. Bei diesem Modell hat $S_{\text {orre }}$ bei allen Drillkorngrenzen für eine bestimmte Temperatur einen festen Wert. Sämtliche Ergebnisse der Monte-Carlo-Simulationen können in einem einzigen Arrhenius-Diagramm dargestellt werden; die freie Energie der Bindung eines Au-Atomes an den Kern der primären Korngrenzversetzung kann ermittelt werden. Bindungsenthalpie und -entropic der Segregation betragen $0,095 \pm 0,01 \mathrm{eV}$ und $k_{\mathrm{B}}(0,49 \pm 0,10)$.
\end{abstract}

\section{INTRODUCTION}

In Part I we presented detailed results of Monte Carlo (MC) simulations of Au segregation, utilizing embedded atom method (EAM) potentials, to [001] twist boundaries in a $\mathrm{Pt}-1$ at. $\% \mathrm{Au}$ alloy in the temperature range $850-1900 \mathrm{~K}$. The approach we have taken utilizes the physical picture that there are state variables that define the thermodynamic state of a planar grain boundary (GB) in a bicrystal $[1,2]$. A total of $6+C$ macroscopic state variables is required to specify the thermodynamic state of a bicrystal- $C$ is the number of chemical components in the alloy; this number of state variables is for a locally relaxed GB [2]. For a single-phase binary alloy bicrystal an eight-dimensional hyperspace is required. Each point in this hyperspace represents a thermodynamic state of a GB. The eight state variables involved are the five macroscopic degrees of freedom of a GB $[3,4]$ temperature, pressure and bulk composition. In our study we fixed four of the five macroscopic degrees of freedom of a [001] twist boundary-as well as the temperature, pressure, and bulk composition -and then systematically varied the twist angle $(\theta)$ between 0 and $45^{\circ}$; because the point group symmetry is $4 \mathrm{~mm}$ this covers all possible 


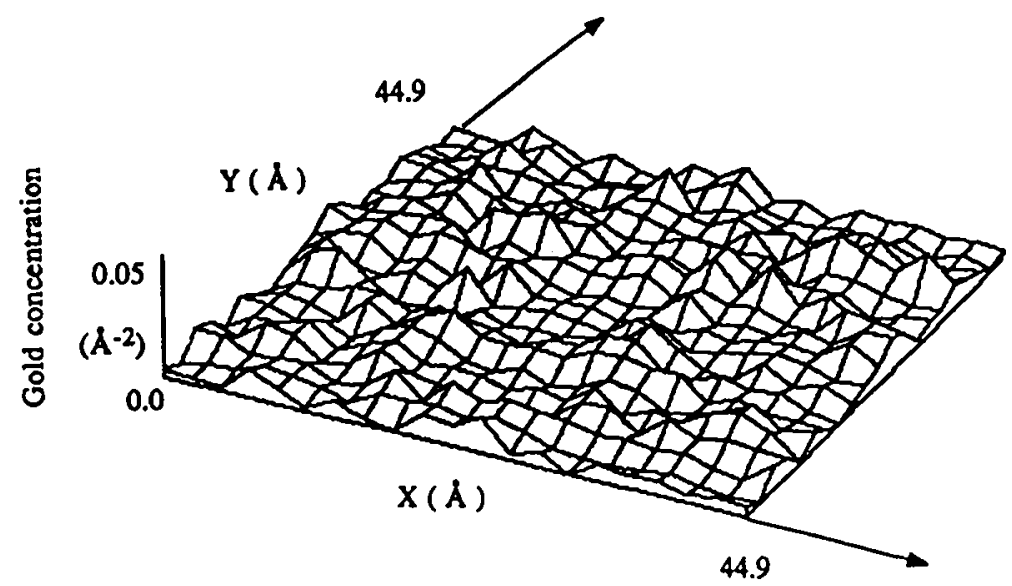

Fig. 1. The spatial distribution of Au atoms-for a $\theta=5^{\circ}[001]$ twist-for the two (002) planes that are immediately adjacent to this interface. The units of concentration are number of $A u$ atoms $\AA^{-2}$. The simulated data are for a total of $5 \cdot 10^{6}$ micro-MC steps. Note very carefully the hill-and-valley topography of the Au atomic density. The valleys correspond to the regions of good atomic fit and the hills to the regions of the cores of the PGBDs.

[001] twist boundaries. In addition, we examined the effects of temperature at a number of different values of fixed $\theta$ between 0 and $45^{\circ}$, while holding pressure, bulk composition and the other four macroscopic degrees of freedom constant. In this manner we explored the chemical composition of each GB, as a function of $\theta$ and temperature; the chemical composition is directly proportional to the interfacial excess concentration of solute and therefore to the Gibbs adsorption isotherm. The MC simulations then give us a detailed atomistic picture of segregation within the context of this thermodynamic framework. In this paper we present a model for the MC simulation results which explains the observed dependencies of the average segregation factor $\left(S_{\text {ever }}\right)$ on temperature and $\theta$-i.e. $S_{\text {aver }}=S_{\text {aver }}(T, \theta)$-in terms of Au segregation to the cores of the orthogonal pairs of primary grain boundary dislocations (PGBDs) that comprise the [001] twist boundaries.

\section{ATOMISTIC MODELS FOR SEGREGATION}

\subsection{A core segregation model}

In Section 5 of Part I we demonstrated that $S_{\text {ver }}$ increases linearly as $\theta$ is increased and it saturates at $\approx 35^{\circ}$, and $S_{\text {aver }}$ also decreases exponentially, as $T$ increases at each value of $\theta ; S_{\text {aver }}$ is a ratio of the $\mathrm{Au}$ concentration in the two (002) planes which adjoin the geometric interface over the bulk value. There is no clear cut dependence of $S_{\text {aver }}$ on the value of $\Sigma$. This result does not agree with the conventional wisdom concerning solute-atom segregation to GBs, which is based on the intuitive idea that solute-atom

tWe exclude from this argument the case of a $\Sigma=3$ coherent twin on a (111) plane which is a special internal interface. Monte Carlo simulations for this case failed to detect any Au segregation above the matrix concentration [?]. segregation to GBs with a better "fit" of the atomic structuret is lower than to general boundaries [5]. MC simulations of segregation to [001] twist boundaries in single phase $\mathrm{Cu}-\mathrm{Ni}$ alloys also demonstrate that this intuitive idea is untenable [6]. In this subsection we present a model which explains the observed dependencies of $S_{\text {aver }}$ on $T$ and $\theta-S_{\text {ver }}=S_{\text {aver }}(T, \theta)$; it is emphasized that the effects of $T$ and $\theta$ are investigated one at a time, therefore the chemical composition of the GB was investigated by varying one state variable while the remaining seven state variables were held constant.

Figure 1 is a three-dimensional display of the distribution of $\mathrm{Au}$ atoms at a twist boundary with $\theta=5.0^{\circ}$. The height of each peak is equal to the density of Au atoms; the units are Au atoms $\AA^{-2}$ at a particular point in the two (002) planes immediately adjacent to the interface, as the results (Fig. 5 of Part I) indicate that Au segregation occurs predominantly at these two planes. The presence of a hill-and-valley type distribution is evident. The valleys correspond to the regions of good atomic fit between the regions of misfit; the hills correspond to the cores of the pairs of orthogonal PGBDs with $b=(a / 2)\langle 110\rangle$. This implies that the lateral Au distribution at the interface is not homogeneous. And that the Au segregation occurs mainly to the cores of the PGBDs. A qualitatively similar result was found for a $\theta=10.4^{\circ}$ boundary. The region of good atomic fit between the cores of the PGBDs is at a somewhat higher Au concentration than the bulk of the bicrystal-see below. (For $\theta>10.4^{\circ}$ the $d$ values between the PGBDs become rather small-see Table 1 in Part I-and it is difficult to distinguish the hills from the valleys in a three-dimensional plot.) We have found for [001] twist boundaries in a $\mathrm{Pt}-3$ at.\% Ni alloy an extremely strong core segregation effect where the hill and valley structure is significantly more pronounced than in this $\mathrm{Pt}-\mathrm{l}$ at. Au alloy 
(Udler and Seidman, unpublished research). Also the above result for this $\operatorname{Pt}(\mathrm{Au})$ system is qualitatively similar to a result for an isolated edge dislocation - that is dissociated in a Ni-10 at. \% $\mathrm{Cu}$ alloywhere the $\mathrm{Cu}$ atoms segregate predominantly to the cores of the partial dislocations, that bound the stacking-fault ribbon, on the expansive side of the slip plane [8]. Also in a study of segregation to $\theta=10.4^{\circ}$, $22.6^{\circ}$ and $36.9^{\circ}(\Sigma=61,13$ or 5$)[001]$ twist boundaries in three $\mathrm{Ni}-\mathrm{Cu}(10,50$ or 90 at.\%) alloys, the $\mathrm{Cu}$ segregation is mainly to a few planes that are adjacent to the interface [6]. The degree of segregation is greatest for the $\mathrm{Ni}$ rich alloys and for the larger twist angles. For the $10.4^{\circ}[001]$ twist boundary, the $\mathrm{Cu}$ concentration has a spatial variation within the boundary plane, though the nature of that variation is different than that found here.

The preceding observations lead us to a model for the results of the MC simulations. We commence by dividing the GB interface into two regions. The first region contains the cores of the PGBDs and the second one is the region of good atomic fit. The structure of the core region is assumed, to first order, to be the same for all $\theta$ 's studied, as the Burgers vector of the PGBDs is identical for all values of $\theta$. Thus, it is logical to assume that the Au concentration at the cores is independent of $\theta$. The average solute-atom concentration at an interface $\left(C_{8}\right)$ can therefore be expressed as the sum of the solute-atom concentrations -i.e. a linear model-in the region of good atomic fit $\left(C_{\mathrm{b}}\right)$ and in the dislocation cores $\left(C_{\text {core }}\right)$

$$
C_{\mathrm{g}}=(1-\gamma) C_{\mathrm{b}}+\gamma C_{\text {core }}, \quad 0<\gamma<1
$$

or

$$
C_{\mathrm{g}}=C_{\text {core }}, \quad \gamma=1
$$

where $\gamma$ is the fraction of atoms at the cores of the PGBDs. The relation between $\gamma$ and $\theta$ in the [001] twist GBs is derived in terms of the dislocation core radius $(\eta)$ and $d-$ equation (2) of Part $\mathrm{I}-$ of the PGBDs. Physically $\gamma$ is the ratio of the area-for the area enclosed by one unit $\left(d^{2}\right)$ of the orthogonal PGBD network - covered by the cores of the PGBDs $\left(4 d \eta-4 \eta^{2}\right)$ divided by $d^{2}$ (Fig. 2). The quantity $\gamma$ is therefore given by

$$
\begin{aligned}
\gamma & =\frac{4 \eta}{d}\left(1-\frac{\eta}{d}\right) \\
& =\frac{8 \eta}{|b|} \sin \left(\frac{\theta}{2}\right)\{1-2 \eta /[|b| \sin (\theta / 2)]\} .
\end{aligned}
$$

$\ddagger$ Equation (3) of Part I fits the MC results using error bars equal to $\pm 2 S D$; hence, we feel justified neglecting the second term in square brackets in equation (4) with respect to unity.

$\S$ For $\eta$ equal to $|\mathrm{b}|$ the "peripheries" of the cores touch when the dislocations are separated by $2|b|$. Thus the Read-Shockley equation yields $\approx 29^{\circ}$ for the geometric condition that the cores overlap when the cylindrical peripheral surfaces just touch along a line.

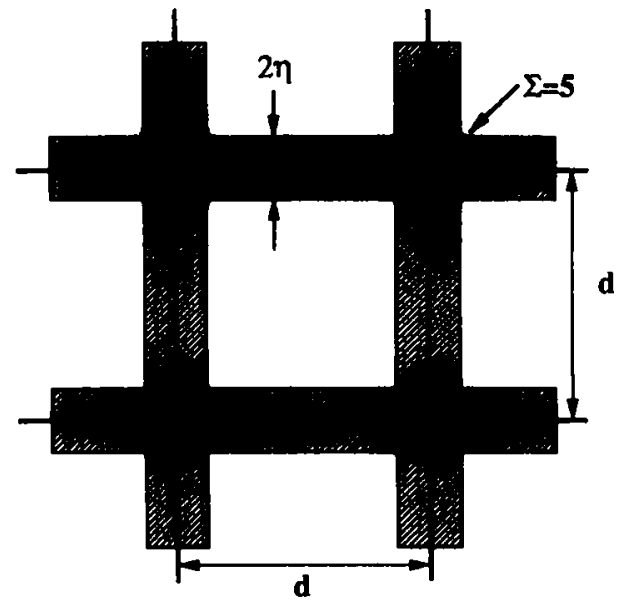

Fig. 2. A schematic diagram illustrating the model used to calculate $\gamma$-the fraction of atoms at the cores of the PGBDs. The quantity $\eta$ is the core radius and $d$ is the interdislocation spacing, as given by the Read-Shockley equation [equation (2) of Part I]. The intersections of the PGBDs correspond to regions of a $\Sigma=5\left(\theta=36.9^{\circ}\right)$ [001] interface.

From the definition of $S_{\text {aver }}$ (Section 5.2 of Part I) we obtain

$$
\begin{aligned}
S_{\text {aver }}(T, \theta)= & 1+\frac{8 \eta}{|\mathbf{b}|} \sin \left(\frac{\theta}{2}\right) \\
& \times[1-(2 \eta /|\mathbf{b}|) \sin (\theta / 2)]\left(S_{\text {core }}-1\right)
\end{aligned}
$$

where $S_{\text {core }}$ is given by $C_{\text {core }} / C_{\mathrm{b}}$-physically this ratio is the segregation enhancement factor for the core region; N.B. that $S_{\text {core }}$ is independent of $\theta$ in this model. $S_{\text {core }}$ has a more direct physical meaning than does $S_{\text {aver }}$, as it takes into account the lateral distribution of solute atoms at the interface. If we can approximately neglect $\ddagger(2 \eta /|\mathbf{b}|) \sin (\theta / 2)$ with respect to unity then equation (4) has the same linear form as equation (3) of Part I, that was derived directly from the MC simulations results exhibited in Fig. 6 of Part I; i.e.

$$
S_{\text {aver }}(T, \theta) \approx 1+(8 \eta /|\mathbf{b}|) \sin \left(\frac{\theta}{2}\right)\left(S_{\text {core }}-1\right) .
$$

The slope $m(T)$ of equation (3) of Part $I$ is now given by

$$
m(T)=(8 \eta /|\mathbf{b}|)\left(S_{\text {core }}-1\right) .
$$

In order, however, to calculate values of $S_{\text {core }}$ from the temperature dependence of $S_{\text {aver }}$ we need to specify a value of $\eta$. The $\mathrm{MC}$ simulations show that the value of $S_{\text {aver }}$ saturates when $\sin (\theta / 2)$ is $\approx 0.3$-this implies $\theta$ is $\approx 35^{\circ}$ when the cores overlap; the saturation effect is particularly clear at low temperatures (850, 900 and $1000 \mathrm{~K}$ )-see Fig. 5 of Part I. Within the context of the PGBD model the cores overlap when the value of $\theta$ increases beyond a certain geometric value. $\$$ For the purpose of evaluating $\eta$ we use the MC value rather than this geometric result, as the latter does not include the atomic relaxations that 
occur at the core; atomic relaxations are a part of this MC simulation (see Section 2 of Part I). Therefore, using the condition $\gamma=1$ at $\sin (\theta / 2) \approx 0.3$ and equation (3) yields

$$
\eta=\frac{|\mathbf{b}|}{4 \sin \left(\frac{\theta}{2}\right)}=0.8|\mathbf{b}| \text {. }
$$

The value $\eta \approx 0.8|b|$ is less than the number $\eta=|b|$ frequently used for the core radius but it is nevertheless a physically reasonable value.

In this model $S_{\text {core }}$ has a single value for all the [001] twist boundaries at a given $T$-i.e. $S_{\text {core }}$ is independent of $\theta$ at each $T$. Figure 3 is an Arrhenius plot of $S_{\text {core }}$; the uncertainties are equal to $\pm 1 S D$ and were obtained from a least squares fit of the calculated values of $S_{\text {core }}$ to a straight line. This plot yields the binding free enthalpy ( $\Delta h_{\text {s-core }}^{\mathrm{b}}$ ) and binding entropy $\left(\Delta s_{\text {t-ore }}^{b}\right)$ of a Au atom to the cores of the PGBDs. The values are $\Delta h_{\text {s-core }}^{\mathrm{b}}=0.095 \pm 0.01 \mathrm{eV}(9.17 \pm$ $0.96 \mathrm{~kJ} \mathrm{~mol}^{-1}$ ) and $\Delta s_{\text {score }}^{\mathrm{b}}=0.49 \pm 0.10 k_{\mathrm{B}}$. The fact that the results fit the classical Langmuir-McLean equation is consistent with the values of $C_{\mathrm{g}}$ calculated-1.2-3.5 at.\% $\mathrm{Au}$. At this level of $\mathrm{Au}$ segregation the two basic assumptions of the simple Langmuir-McLean model, the absence of soluteatom interactions at the interface and a single value of $\Delta h_{\text {score }}^{\mathrm{b}}$, are approximately satisfied. Thus for the case of [001] twist boundaries the slightly oversized Au atoms sit at substitutional sites in the cores of the dislocations (this is observed directly via the $\mathrm{MC}$ simulations) and equation (6) of Part I defines the $T$ dependence of the Gibbs binding free energy. Using the latter definition the expression for $\Delta g_{\text {i-core }}^{b}$ for $A u$ atom segregation to [001] twist boundaries is given by

$$
\begin{aligned}
\Delta g_{\text {ecore }}^{\text {b }}(T)= & {[(0.095 \pm 0.01)} \\
& \left.-k_{\mathrm{B}} T(0.49 \pm 0.10)\right] \mathrm{eV} \cdot \text { atom }^{-1}
\end{aligned}
$$

It is emphasized that equation ( 7 ) is for $\eta \approx 0.8|\mathbf{b}|$, and if $\eta$ is less than this value then this $\Delta g_{\text {roore }}^{b}(T)$ represents a lower bound to $\Delta h_{2-\mathrm{bb}}^{\mathrm{b}}$. A small decrease in $\eta$ results in a small increase in $\Delta g_{\text {wore }}^{b}$, and a concomitant increase in $S_{\text {core }}$, such that it approaches a saturation value at each $T$. If the latter obtains the region of good atomic fit at the interface has approximately the same Au concentration as the bulk value.

\subsection{The question of other possible core segregation models}

In the previous subsection we considered a specific model for Au segregation to the cores of the PGBDs. And therefore the question arises-is there another model which also explains the data? A second possible model is that the Au atoms segregate mainly to the regions of intersection of the cores of the PGBDs; the intersections of the PGBDs have the structure of a $\Sigma=5[001$ ] twist boundary (Fig. 2) [9]. Thus as $\theta$ increases the fractional area of the interface covered

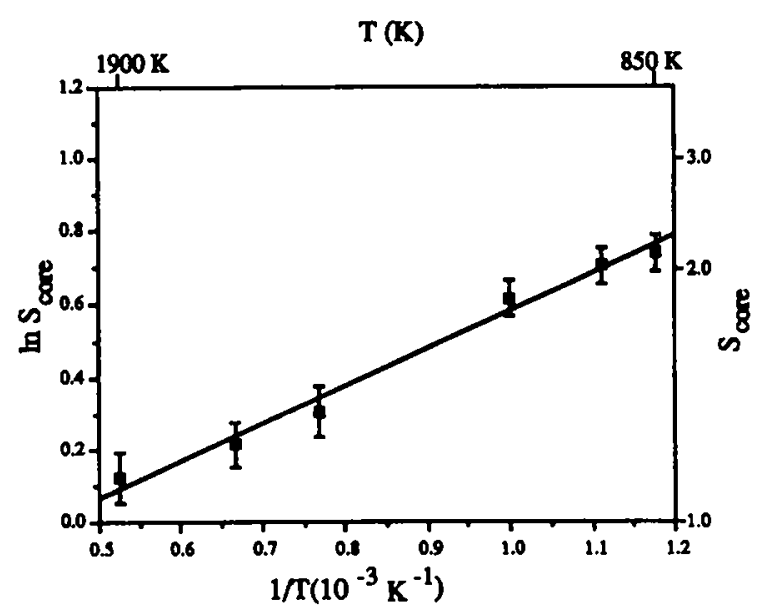

Fig. 3. An Arrhenius plot of $S_{\text {core }}$. The $S_{\text {core }}$ values were deduced from the results in Fig. 6 of Part I employing equation (5) with the core radius ( $\eta$ ) equal to $0.8|\mathrm{~b}|$, where $|b|$ is the magnitude of the Burgers vector. Each error bar is equal to \pm one standard deviation, and was obtained from a least squares fit of the values of $S_{\text {core }}$ to a straight line.

by square patches of the $\Sigma=5$ structure increases. In this case $\gamma$ in equation (3) is given by

$$
\gamma=\frac{\eta^{2}}{d^{2}}=\frac{4 \eta^{2}}{b^{2}} \sin ^{2}\left(\frac{\theta}{2}\right)
$$

thus

$$
\begin{aligned}
C_{\mathrm{s}} & =(1-\gamma) C_{\mathrm{b}}+\gamma C_{\text {core }} \\
& =C_{\mathrm{b}}+\left(C_{\text {core }}-C_{\mathrm{b}}\right)\left(4 \eta^{2} / \mathrm{b}^{2}\right) \sin ^{2}\left(\frac{\theta}{2}\right)
\end{aligned}
$$

and

$$
S_{\text {ever }}=1+\left(S_{\text {core }}-1\right)\left(4 \eta^{2} / b^{2}\right) \sin ^{2}\left(\frac{\theta}{2}\right)
$$

This second model predicts that $S_{\text {aver }}$ should be a function of $\sin ^{2}(\theta / 2)$ and not $\sin (\theta / 2)$, as the linearized form of equation (4) of subsection 2.1 predicts; N.B. the MC simulation data is linear up to $\sin (\theta / 2) \approx 0.3$. A generalization of these two extreme models yields an equation of the form

$$
S_{\mathrm{aver}}=1+\lambda \sin ^{6}\left(\frac{\theta}{2}\right)
$$

where $\zeta$ is an exponent in the range $1 \leqslant \zeta \leqslant 2$, and $\lambda$ is a constant containing $S_{\text {core }}$. Our MC simulations are well approximated by $\zeta=1$ up to $\sin (\theta / 2) \approx 0.3$; after this value $C_{8}$ is approximately a constant-see Part I. Thus the model of subsection 2.1 is a reasonable explanation of the results obtained, and there is no need to use this second model.

\subsection{The role played by elastic interactions in grain boundary segregation}

The classical picture of solute-atom segregation to individual dislocations or to grain boundaries 
comprised of arrays of dislocations has relied heavily on the use of the linear theory of elasticity [10-12]. Although it has been pointed out that the core term could certainly be the dominant one for solute atomdislocation interactions [13]. We have considered the linear elastic interactions between a solute atom and a twist boundary using isotropic elasticity theory in some detail in order to assess their physical signifcance [14]; the equations are quite general and cover all possible solute and solvent atoms in the periodic table within the context of a linear elastic model. The first interaction arises from a size misfit effect between the solute and solvent atoms; the misfit energy is given by $E_{\text {mis }}=p \Delta V[10-12,15-20]$. The second elastic interaction is due to the volume expansion around a screw dislocation and the resulting hydrostatic stress $[17,18,21]$. The third elastic interaction is due to the differences between the shear and bulk moduli of the solute and solvent atoms respectively-this is commonly called the inhomogeneity effect $[10-12,15-17,19,20]$. The misfit factor is given by:

$$
\epsilon_{R}=\frac{R_{\text {imp }}-R}{R}
$$

where $R_{\text {imp }}$ and $R$ are the atomic radii of the solute and solvent atoms. While the inhomogeneity effect is characterized by

$$
\epsilon_{K}=\frac{K_{\mathrm{imp}}-K}{K} \text { and } \epsilon_{\mu}=\frac{\mu_{\mathrm{imp}}-\mu}{\mu}
$$

where $\mu_{\text {imp }}, K_{\text {imp }}, \mu, K$ are the shear and bulk moduli of the solute and solvent atom respectively.

In the case of a pure twist boundary the interaction energy between a solute atom and an array of screw dislocations due to the $p \Delta V$ effect is zero since the stress field associated with a twist boundary is pure shear-i.e. there is no hydrostatic component $p$. Second, the interaction energy between a $\mathrm{Au}$ solute atom and a twist boundary due to a volume expansion around each screw dislocation and the resulting hydrostatic stress field is a repulsive interaction for the $\operatorname{Pt}(\mathrm{Au})$ system since $\mathrm{Au}$ has a slightly larger atomic radius than a $\mathrm{Pt}$ atom; the ionic radii of $\mathrm{Au}$ and $\mathrm{Pt}$ are 0.144 and $0.139 \mathrm{~nm}$, respectively. Third, the inhomogeneity interaction is attractive and therefore this effect alone predicts that $\mathrm{Au}$ segregation should occur in the $\operatorname{Pt}(\mathrm{Au})$ system. However, when both interactions are summed the net effect is a repulsive interaction for this system at high angles. At small twist angles there is a net attractive interaction energy along the dislocation lines, and a repulsive interaction where the dislocations cross. This result is in contrast to the Monte Carlo results which exhibit increasing solute segregation with increasing twist angle. Further considerations concerning the interactions between solute atoms and grain boundaries within the context of isotropic elasticity theory are considered elsewhere in detail [14].

\section{SUMMARY AND CONCLUSIONS}

1. A three-dimensional plot (Fig. 1) of the density of Au atoms $\AA^{-2}$ vs the $x$ and $y$ position coordinates for the (002) planes, immediately adjacent to the interface, for a $\theta=5^{\circ}[001]$ twist boundary reveals the presence of a hill-and-valley structure in the Au segregation behavior, the temperature is $850 \mathrm{~K}$. The valleys correspond to the regions of good atomic fit between the regions of misfit; the hills correspond to the cores of the pairs of orthogonal PGBDs with $\mathbf{b}=(a / 2)\langle 110\rangle$. This suggests that the lateral $\mathrm{Au}$ distribution at the interface is not homogeneous, and the Au segregation occurs mainly substitutionally to the core regions. A qualitatively similar result was found for a $\theta=10.4^{\circ}$ [001] twist boundary. The region of good atomic fit between the core regions is at a somewhat higher $\mathrm{Au}$ concentration than the bulk of the bicrystal.

2. The preceding observations are the basis of a model for the MC simulations. In this model we divide the $\mathrm{GB}$ interface into two regions. The first region contains the cores of the PGBDs and the second one is the region of good atomic fit-see Fig. 2. The structure of the core region is taken to be the same for all $\theta$ 's studied, as the Burgers vector of the PGBDs is identical for all values of $\theta$. The average Au concentration at an interface $\left(C_{\mathrm{g}}\right)$ is expressed as the sum of the $\mathrm{Au}$ concentrations in the region of good atomic fit $\left(C_{\mathrm{b}}\right)$ and in the cores of the dislocations $\left(C_{\text {core }}\right)$. This leads to the following linearized expression for $S_{\text {aver }}$

$$
S_{\text {aver }}(T, \theta) \approx 1+(8 \eta /|\mathbf{b}|) \sin \left(\frac{\theta}{2}\right)\left(S_{\text {core }}-1\right) .
$$

From this equation $S_{\text {core }}\left(S_{\text {core }}=C_{\text {core }} / C_{\mathrm{b}}\right)$ is calculated for a core radius $(\eta)$ equal to $0.8|\mathrm{~b}|$; this $\eta$ corresponds to the value of $\theta$ at which the cores overlap; this occurs at $\sin (\theta / 2) \approx 0.3$, as observed in the MC simulations.

3. In the context of the above model $S_{\text {core }}$ has a single value for all twist boundaries at a given $T$. Figure 3 is an Arrhenius plot of $S_{\text {core }}$. From this plot the following explicit expression for the Gibbs binding free energy of a $A u$ atom to the cores $\left(\Delta g_{\text {score }}^{\mathrm{b}}\right)$ of PGBDs in [001] twist boundaries is obtained

$$
\begin{aligned}
\Delta g_{\text {s-core }}^{\mathrm{b}}(T)=[( & 0.095 \pm 0.01) \\
& \left.-k_{\mathrm{B}} T(0.49 \pm 0.10)\right] \mathrm{eV} \cdot \text { atom }^{-1}
\end{aligned}
$$

This expression is for $\eta \approx 0.8|\mathbf{b}|$; if $\eta$ is less than this value then this $\Delta g_{\text {-core }}^{\mathrm{b}}(T)$ represents a lower bound.

4. A summary of the basic physical picture that emerges from these MC simulations is as follows. At a constant temperature the average segregation factor $\left(S_{\text {aver }}\right)$ increases as the twist angle $(\theta)$ increases and it saturates at $\theta \approx 35^{\circ}$. The value of $S_{\text {aver }}$ increases linearly to $\theta \approx 35^{\circ}$, and is then approximately independent of $\theta$. The $\mathrm{MC}$ simulations indicate that the $\mathrm{Au}$ segregation occurs primarily substitutionally to the cores of the PGBDs, and therefore the 
Au concentration at the interface depends on the fraction of atoms in the bicrystal that are located in the cores. This explains why the value of $S_{\text {aver }}$ is function of $\theta$ and why it saturates when the cores of the PGBDs overlap. When the cores overlap essentially all of the atoms at the interface are in the cores of the PGBDs and increasing $\theta$ does not change significantly the fraction of sites in the bicrystal at the cores of the dislocations. Evidence for very strong $\mathrm{Ni}$ atom segregation to the cores of screw dislocations in a [001] twist boundaries has been bound in a $\mathrm{Pt}-3$ at. \% $\mathrm{Ni}$ alloy via Monte Carlo simulations (Udler and Seidman, unpublished research).

5. The three possible linear elastic interactions between a twist boundary and a gold atom were considered in detail [14]. First, the interaction between a solute atom and a twist boundary is zero since its stress field is pure shear; i.e. there is no hydrostatic component. Second, the interaction energy between a Au atom and a twist boundary is due to a volume expansion around each screw dislocation and the resulting hydrostatic stress was shown to be a repulsive interaction for the $\mathrm{Pt}(\mathrm{Au})$ system since $\mathrm{Au}$ has a larger atomic radius than a $\mathrm{Pt}$ atom. Third, the inhomogeneity interaction is attractive. When, however, both interactions are summed the net effect is a repulsive interaction for this system at high angles. Although the tendency for segregation increases as the interdislocation spacing decreases. This is in contrast to the Monte Carlo results which show increasing segregation with decreasing interdislocation spacing. The isotropic elasticity theory results are discussed elsewhere [14].

Acknowledgements - This research is supported by the NSF (grant No. DMR-8819074-Dr B. MacDonald, grant officer). It made use of central facilities supported by the NSF through Northwestern's Materials Research Center, Grant No. DMR 85-20280. The simulations were carried out at the National Center for Supercomputing Applications at the University of Illinois at Urbana-Champaign on the CRAY X-MP/48 system. Mr A. Seki was kindly supported by Sumitomo metal Industries, LTD., Osaka, Japan. Dr S. M. Foiles is supported by the U.S. Department of Energy, Office of Basic Energy Sciences, Division of Materials Science. We thank Dr M. I. Baskes and Professor A. J. Freeman for their kind interest in this research program; Dr K. L. Merkle and Dr D. Udler for stimulating discussions; and Drs G. Martin, B. Legrand and V. Pontikis for stimulating discussions (made possible by NSF International Grant No. INT-8715232-Dr C. Glenday, grant officer-to DNS).

\section{REFERENCES}

1. E. W. Hart, Scripta metall. 2, 179 (1968); E. W. Hart, in The Nature and Behavior of Grain Boundaries (edited by Hsun Hu), p. 155. Plenum Press, New York (1972).

2. J. W. Cahn, J. Physique, Coll. 43, C6-199 (1982).

3. W. T. Read Jr and W. Shockley, Phys, Rev. 78, 275 (1950).

4. W. T. Read Jr, Dislocations in Crystals. McGraw-Hill, New York (1953).

5. R. W. Balluffi, in Interfacial Segregation (edited by
W. C. Johnson and J. M. Blakely), p. 193. Metals Park, Ohio (1979).

6. S. M. Foiles, Phys. Rev. B 40, 11502 (1989).

7. M. Ueno, A. Seki, Y. Oh and D. N. Seidman. To be published.

8. S. M. Foiles, Mater. Res. Soc. Symp. Proc. 63, 61 (1985).

9. D. Schwartz, V. Vitek and A. P. Sutton, Phil. Mag. A 51, 499 (1985).

10. S. Amelinckx and W. Dekeyser, Solid St. Phys. 8, 405 (1959).

11. J. C. M. Li, J. appl. Phys. 31, 1318 (1960); J. C. M. Li, Acta metall. 9, 384 (1961); J. C. M. Li, in Electron Microscopy and Strength of Crystals (edited by G. Thomas and J. Washburn), pp. 734-736. Interscience, New York (1963).

12. J. P. Hirth and J. Lothe, Theory of Dislocations, pp. 462-473. McGraw-Hill, New York (1968); idem, ibid, 2nd edn, pp. 512-514, 670-672. Wiley, New York (1982).

13. H. Reiss, J. chem. Phys. 40, 1783 (1964).

14. D. Udler and D. N. Seidman, submitted for publication (1991).

15. A. H. Cottrell and B. A. Bilby, Proc. R. Soc. A 62, 49 (1949); B. A. Bilby, Proc. Phys. Soc. A 61, 191 (1950); A. H. Cottrell, Dislocations and Plastic Flow in Crystals, pp. 56-57, 134-136. Oxford Univ. Press (1953).

16. J. D. Eshelby, Phil. Trans. R. Soc. A 244, 87 (1951); idem, Solid St. Phys. 3, 79 (1965).

17. R. L. Fleischer, Acta metall. 9, 996 (1961).

18. I. Saxl, Czech. J. Phys. B14, 381 (1964).

19. J. Friedel, Dislocations. Addison-Wesley, Reading, Mass. (1964).

20. J. D. Eshelby, in The Physics of Metals (edited by P. B. Hirsch), Vol. 2, pp. 33-42. Cambridge Univ. Press (1975).

21. H. Stehle and A. Seeger, Z. Phys. 146, 217 (1956).

22. M. P. Allen and D. J. Tildesley, Computer Simulation of Liquids. Clarendon Press, Oxford (1987).

23. R. Friedberg and J. E. Cameron, J. chem. Phys. 52, 6049 (1970).

24. G. Jacucci and A. Rahman, Nuovo Cimento D4, 341 (1984).

\section{APPENDIX A}

\section{Statistical Errors in Monte Carlo Simulations}

We review the calculation of the variance in the mean value of a specified property $(\langle P\rangle)$ and the error in $\langle P\rangle$ for an $M C$ simulation [22]; this assumes Gaussian statistics. Consider an MC simulation run consisting of $\tau_{\text {run }}$ micro-MC steps. The $\langle P\rangle$ for a run is

$$
\langle P\rangle_{\text {nun }}=\frac{1}{\tau_{\text {run }}} \sum_{\tau=1}^{\text {rnon }} P(\tau)
$$

i.e. the sum is over all the micro-MC steps. If the value of each quantity $P(\tau)$ is statistically independent of all the other values of this same quantity, then the variance of the mean is given by

$$
\sigma^{2}\left(\langle P\rangle_{\text {run }}\right)=\sigma^{2}(P) / \tau_{\text {rud }}
$$

where

$$
\sigma^{2}(P)=\left\langle\delta P^{2}\right\rangle_{\text {run }}=\frac{1}{\tau_{\text {run }}} \sum_{i=1}^{r_{\text {ren }}}\left[P(\tau)-\langle P\rangle_{\text {run }}\right]^{2} .
$$

The estimated error in $\langle P\rangle$ is the square root of $\sigma^{2}\left(\langle P\rangle_{\mathrm{run}}\right)$. The data points obtained, however, in an MC simulation are usually not independent, as we store configurations with a frequency such that they are highly correlated with one another. It is necessary to build into equation (A2) the fact that these correlations last for a number of micro-MC steps. 
In the presence of correlations we have $[23,24]$

$$
\sigma^{2}\left(\langle P\rangle_{\text {run }}\right)=s \sigma^{2}(P) / \tau_{\text {run }}
$$

where $s$ is the "statistical inefficiency." The value of $s$ is unknown before the simulation is made and it must be calculated at the end of each run. The definition [22] used for $s$ is

$$
s \equiv \lim _{\mathrm{T} \rightarrow \boldsymbol{x},} \frac{\tau_{b} \sigma^{2}\left(\langle P\rangle_{b}\right)}{\sigma^{2}(P)}
$$

where $\tau_{b}$ is a block which contains a specified number of micro-MC steps (the size is chosen by the investigator). The total length of a run is divided into $n_{b}$ blocks of length $\tau_{b}$, such that $n_{b} \tau_{b}=\tau_{\text {run }}$. The mean value of each block is then determined and they are used to calculate the variance of $\langle P\rangle_{b}$ from the following equation

$$
\sigma^{2}\left(\langle P\rangle_{b}\right)=\frac{1}{n_{b}} \sum_{b=1}^{n_{b}}\left(\langle P\rangle_{b}-\langle P\rangle_{\text {run }}\right)^{2} .
$$

The value of $\sigma^{2}\left(\langle P\rangle_{b}\right)$ is inversely proportional to $\tau_{b}$ for large values of $\tau_{b}$, as the blocks become large enough to be statistically uncorrelated. The quantity $s$ is the limiting ratio of the observed variance to the limit expected on the basis of an uncorrelated Gaussian distribution. The value of $s$ is determined by plotting the value of the right hand side of equation (A5) against $\left(\tau_{b}\right)^{1,2}$. Figure $A 1$ is an illustrative example of this statistical analysis for the total electronic potential energy; the values of $s$ and $\tau_{b}$ are in macro-MC steps; one macro-MC step is arbitrarily defined to be equal to 400 micro-MC steps atom ${ }^{-1}$ for the purpose of plotting Fig. A1. The data exhibited in Fig. Al has an asymptotic

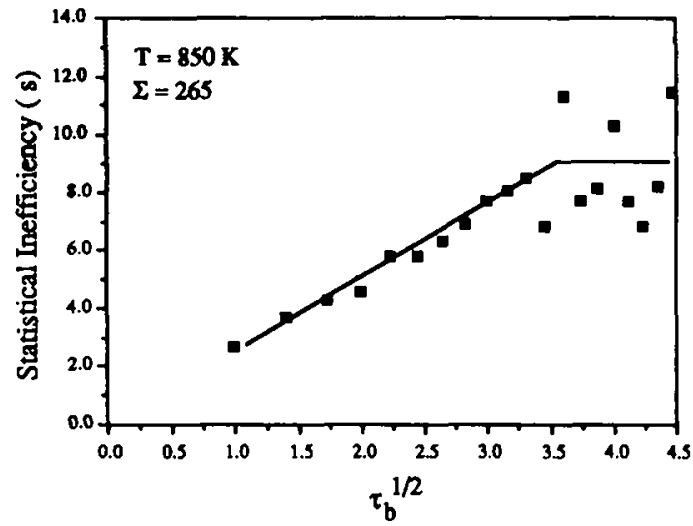

Fig. Al. A plot of the statistical inefficiency (s) vs $\tau_{b}^{1 / 2}$, where $\tau_{b}$ is the block size; both $s$ and $\tau_{b}$ are in macro-MC steps; one macro-MC step equals 400 micro-MC steps atom $^{-1}$. The data in this figure is for the total electronic potential energy $\left(E_{\mathrm{to}}\right)$ of a $5.0^{\circ}[001]$ twist boundary at $850 \mathrm{~K}$

value of $\approx 10$. The value of $\left\langle E_{\text {tot }}\right\rangle$ is equal to $-29,754.2 \mathrm{eV}$ [equation (A1)], and the value of $\sigma^{2}\left(E_{\text {tol }}\right)$ is equal to 136 $(\mathrm{eV})^{2}$ [equation (A3)]; therefore

$$
\sigma^{2}\left(\left\langle E_{\mathrm{tot}}\right\rangle_{\mathrm{run}}\right)=(10 / 400) \sigma^{2}\left(E_{\mathrm{tot}}\right)=3.4(\mathrm{eV})^{2}
$$

so $\sigma\left(\left\langle E_{\text {tot }}\right\rangle_{\text {run }}\right)= \pm 1.8 \mathrm{eV}$; thus, the accuracy is $( \pm 1.8 / 29,754) 10^{2}=6.05 \cdot 10^{-3} \%$ for $E_{\text {tot }}$ for this run. 